\title{
The outpatient assessment of patients with anemia by a general internal medicine service
}

\author{
H. McFadgen*1, S. Couban², S. Doucette ${ }^{3}$, A. Kreuger-Naug ${ }^{4}$, S. Shivakumar ${ }^{2}$ \\ ${ }^{1}$ Dalhousie University Faculty of Medicine, Dalhousie University, Halifax, Nova Scotia, Canada \\ ${ }^{2}$ Division of Hematology, Department of Medicine, Dalhousie University and Nova Scotia Health Authority, Halifax, Nova Scotia, \\ Canada \\ ${ }^{3}$ Research Methods Unit, Nova Scotia Health Authority, Halifax, Nova Scotia, Canada \\ ${ }^{4}$ Division of General Internal Medicine, Department of Medicine, Dalhousie University and Nova Scotia Health Authority, Halifax, \\ Nova Scotia, Canada
}

Received: April 6, 2017

DOI: $10.5430 /$ jha.v6n3p41
Accepted: May 7, 2017

Online Published: May 11, 2017

\begin{abstract}
At the Queen Elizabeth II Health Sciences Centre in Halifax, Nova Scotia, 2,400-2,800 new outpatient referrals for hematology consultation are received annually and approximately $10 \%$ of these referrals are specifically for isolated anemia. In recent years, such referrals have been sent from hematology to general internal medicine (GIM) for assessment and management. A retrospective chart review was conducted of a cohort of 99 patients from 2013 to describe the demographics, assessment, management and outcome of these patients, as well as to inform whether this practice should continue. The median age of patients was 60.3 years ( $\min 19.4$, max 97.6) and $62 \%$ were female. The median hemoglobin level was $109.0 \mathrm{~g} / \mathrm{L}(\min 66$, $\max 137)$ at the time of referral and the median wait time was 53 days (min 8 days, max 171 days). Pearson's correlation analysis revealed that those with lower hemoglobin levels were seen more quickly. The patients had an additional 2.8 comorbidities on average, and were significantly more likely to receive non-anemia related adjustment to care with increasing number of comorbidities. A small proportion of patients $(n=5,5.1 \%)$ were referred from GIM back to hematology, whereas $21 \%$ were referred to gastroenterology. A small number of patients $(n=5,5.1 \%)$ underwent a bone marrow aspirate and biopsy. The most common diagnoses identified in the initial clinic letters were iron deficiency anemia $(n=59,59.6 \%)$ and anemia of chronic disease $(n=8,8.1 \%) .26 .3 \%$ did not have a diagnosis identified. These findings support our practice to have patients with an isolated anemia evaluated by a general internist rather than a hematologist. Most of these patients had iron deficiency anemia or the anemia of chronic disease and received additional care for their comorbid conditions in the GIM clinic. Further work will help to define how such patients can be most effectively assessed and treated.
\end{abstract}

Key Words: Anemia, Hematology, General internal medicine, Outpatient, Referral, Management

\section{INTRODUCTION}

Anemia is the most common blood disorder, with a significant impact on morbidity and mortality. ${ }^{[1]}$ In an analysis of global anemia burden, Kassebaum et al. ${ }^{[2]}$ demonstrated that iron-deficiency anemia (IDA) is the most common cause of anemia globally, followed by a number of infectious disease etiologies (particularly in developing countries), while primary hematologic disorders account for a relatively small

*Correspondence: H. McFadgen; Email: Heather.McFadgen@Dal.Ca; Address: Dalhousie University Faculty of Medicine, Dalhousie University, Halifax, Nova Scotia, Canada. 
percentage of the overall cases of anemia. Aside from those with chronic kidney disease (CKD), the analysis excluded individuals with known chronic illnesses, suggesting that the overall contribution of isolated hematologic disorders may have been overestimated. Given this, the question arises as to which health providers are most appropriate to initially investigate, treat and follow patients found to have anemia.

Subspecialty care has been compared to generalist care in the setting of many chronic illnesses, but there are no studies in the literature specifically addressing care of patients with anemia. While it has been postulated that patients with particular illnesses have improved clinical outcomes and adherence to treatment guidelines when cared for by a subspecialist as compared to a generalist, ${ }^{[3,4]}$ the association has not always been shown to be a strong one. ${ }^{[5]}$ Some suggest that patients with chronic illnesses may be most effectively cared for through co-management by both generalists and subspecialists. ${ }^{[6]}$ In a systematic review of the literature on this topic, Smetana et al. ${ }^{[7]}$ found that in studies where subspecialty care was favoured, selection bias and relevant confounders (practice environment, physician experience, etc.) were often not adequately addressed, leading to further confusion in the specialist versus generalist debate.

At the Queen Elizabeth II Health Sciences Centre in Halifax, Nova Scotia, approximately 2,400-2,800 new outpatient referrals for hematology consultation are received each year, and about $10 \%$ of these referrals are specifically for an isolated anemia. Since isolated anemia is rarely caused by a primary hematologic disease, we adopted a practice of directing outpatient referrals for assessment of anemia to General Internal Medicine (GIM) specialists. This study will describe the assessment, management and outcome of patients triaged to GIM by hematology, to determine whether this practice should continue. This project will also contribute to the existing body of literature surrounding which specialities are most appropriate to care for patients with specific medical diagnoses.

\section{Methods}

\subsection{Data source and demographic variables}

A retrospective chart review was conducted of all ambulatory patients whose referrals for an isolated anemia were sent to GIM from hematology in 2013. Clinic letters and correspondence documents for consecutive patients referred to hematology and redirected to GIM in 2013 were accessed through the Nova Scotia Health Authority's Horizon Patient Folder (HPF) system. Patients who also had other cytopenias (thrombocytopenia or leukopenia) or who were referred to hematology for reasons other than anemia were excluded. Data on each patient's first appointment was accessed from the year 2013, and a follow up period of up to eighteen months was reviewed. The patient cohort was characterized using summary statistics and included median age, as well as geographical distribution of patients. Sex was calculated as a percentage of the total study population.

\subsection{Analysis of the referral process}

Analysis of the referral process was performed by calculating the median length of time between receipt of referral by hematology and the initial GIM appointment, as well as by a Pearson's correlation between anemia severity and time to initial appointment. Due to unavailability of data, we were unable to assess the time between the referral arriving in hematology and subsequently being sent to GIM. However, the process by which referrals are sent from hematology to GIM is systematic and consistent, such that we do not believe this omission detracts from the analysis of the referral process. Anemia severity was classified based on World Health Organization (WHO) criteria, where non-anemia is a hemoglobin of $>130 \mathrm{~g} / \mathrm{L}$ or $>120 \mathrm{~g} / \mathrm{L}$ for men and non-pregnant women, respectively; mild anemia is a hemoglobin of 110-129 g/L or 110-119 g/L for men and non-pregnant women, respectively; moderate anemia is $80-109 \mathrm{~g} / \mathrm{L}$ for both men and non-pregnant women, and severe anemia is a hemoglobin of $<80$ for both men and non-pregnant women. Baseline hemoglobin was defined as the hemoglobin level at the time of referral, and was summarized in conjunction with baseline white blood cell (WBC) count and platelet (PLT) count. Hemoglobin level at the time of the first GIM appointment was also summarized. Type of anemia was categorized as either microcytic, normocyctic or macrocytic, as determined by the red blood cell (RBC) mean corpuscular volume (MCV) in accordance with accepted laboratory parameters at the Queen Elizabeth II Health Science Centre.

\subsection{Outcome of referral}

Simple statistics were used to describe the frequency with which a diagnosis was identified at the initial GIM appointment as well as the percentage of those diagnosed with iron deficiency anemia, anemia of chronic disease or other. Investigations measured included the number of blood tests ordered and frequency of bone marrow biopsies performed. 61 patients had sufficient follow-up data in the 18-month period to be included in a sub-group analysis of patient outcome, defined as a change in the category of anemia severity as outlined above. Frequency of re-referral to hematology, as well as referral to gastroenterology (GI) or gynecology was calculated as a percentage of the total study population, in addition to being correlated with the type of anemia (microcytic, macrocytic or normocytic). The mean number of 
comorbidities was evaluated from the clinic letters of each patient. The number of comorbidities was enumerated and correlated with whether or not the patient received any nonanemia related adjustment of care (e.g., changes to blood pressure medication). Frequency of follow-up appointments in the year following the initial appointment was calculated as a percentage of the total study population.

\section{Results}

The characteristics of the study population are summarized in Table 1. The median time from referral to being seen by GIM was 53 days (min 8 days, max 171 days), a period of time that decreased significantly as severity of anemia increased (see Table 2). The majority of patients were found to have a normocytic anemia (48\%), $43.9 \%$ had a microcytic anemia, and $8.2 \%$ had a macrocytic anemia. The most common diagnosis in this study population as identified in the initial GIM clinic letter, was IDA (59.6\%), with $8.1 \%$ being categorized as anemia of chronic disease, $26.3 \%$ having no identified diagnosis, and $6.1 \%$ having another cause identified (malignancy, B12 deficiency, medication effect, etc.). The largest proportion of patients $(38.4 \%$ ) had between four and ten blood tests ordered at the initial appointment, and $5.1 \%(\mathrm{n}=5)$ had bone marrow biopsies performed. $5.1 \%$ $(n=5)$ of patients were re-referred to hematology, $21.4 \%$ were referred to GI and $4.1 \%$ were referred to gynecology.
The majority of patients referred to GI had a microcytic anemia (see Table 3). The mean number of comorbidities per patient was 2.8, and the occurrence of non-anemia related adjustment to care increased significantly with increasing number of comorbid diseases (see Table 4). Of the 61 patients included in the sub-group analysis of patient outcome , $54.1 \%$ had an improvement in their hemoglobin that brought them up a severity level, $39.3 \%$ stayed in the same severity category, and $6.6 \%$ dropped to a worse severity category, eighteen months following the initial consult.

Table 1. Characteristics of patients and the anemia

\begin{tabular}{ll}
\hline & Median (IQR) \\
\hline Age & $60.3[46.2-71.7]$ \\
Hgb at time of referral & $109.0[97.0-123.0]$ \\
WBC at time of referral & $7.2[5.7-8.2]$ \\
PLT at time of referral & $259.0[210.5-320.5]$ \\
Hgb at time of first appointment & $114.0[100.3-125.8]$ \\
Number of comorbidities & $2.0[1.0-4.0]$ \\
Time from receipt of referral to first visit (days) & $53.0[36.5-85]$ \\
\hline & Frequency (\%) \\
\hline Sex & $37(37.4)$ \\
- Male & $62(62.6)$ \\
- Female & \\
Type of anemia & $43(43.9)$ \\
- Microcytic & $47(48)$ \\
- Normocytic & $8(8.2)$ \\
\hline
\end{tabular}

Table 2. Correlation between anemia severity and time to be seen

\begin{tabular}{lccl}
\hline \multirow{2}{*}{ Anemia Severity } & \multicolumn{2}{c}{ Time to be seen (days) } & \\
\cline { 2 - 3 } & Mean $($ SD) & Median (IQR) & \\
\hline Normal $(\mathrm{n}=15)$ & $87.1(42.9)$ & $91[53-129]$ & Pearson's Correlation $=0.43$ \\
Mild $(\mathrm{n}=33)$ & $67.6(26.7)$ & $63[48-86]$ & $p<.0001$ \\
Moderate $(\mathrm{n}=42)$ & $57.1(36.7)$ & $49[27-79]$ & \\
Severe $(\mathrm{n}=8)$ & $30.4(12.7)$ & $32[20-42]$ & \\
\hline
\end{tabular}

Table 3. Specialty referral by type of anemia

\begin{tabular}{lllll}
\hline Type of anemia & Referred to hematology & $\boldsymbol{p}$-value & Referred to GI & $p$-value \\
\hline Microcytic $(\mathrm{n}=42)$ & $1(2.4 \%)$ & .59 & $15(35.7 \%)$ & .006 \\
Normocytic $(\mathrm{n}=47)$ & $4(8.5 \%)$ & & $4(8.5 \%)$ & \\
Macrocytic $(\mathrm{n}=8)$ & $0(0 \%)$ & & $2(25.0 \%)$ & \\
\hline
\end{tabular}

Table 4. Non-anemia adjustment to care by number of comorbidities

\begin{tabular}{lll}
\hline $\begin{array}{l}\text { Number of } \\
\text { comorbidities }\end{array}$ & $\begin{array}{l}\text { Non anemia related } \\
\text { adjustment of care }\end{array}$ & $\boldsymbol{p}$-value \\
\hline $0(\mathrm{n}=14)$ & $1(7.1 \%)$ & \\
$1-2(\mathrm{n}=36)$ & $11(30.6 \%)$ & .0005 \\
$3-5(\mathrm{n}=37)$ & $20(54.1 \%)$ & \\
$>5(\mathrm{n}=12)$ & $8(66.7 \%)$ & \\
\hline
\end{tabular}

Published by Sciedu Press

\section{Discussion}

In this study, we demonstrate that patients with an isolated anemia are appropriately and effectively assessed and managed in a GIM clinic. Most such patients have iron deficiency anemia and underwent further referral to GI and gynecology services. Four major findings stand out for consideration of the appropriateness of this practice, including: the effectiveness of the referral process for identifying patients with the 
most severe anemia and triaging appropriately, the practice of the general internist to provide more generalized care in addition to working up the anemia, the low rate of re-referral to hematology and relative higher rate of referral to GI, and finally the low number of patients who required bone marrow biopsy as part of their work-up.

During the last decade, Canada has seen many initiatives to reduce patient wait-times for medical services. Excessively long wait times have a range of negative effects on the patients' health, as well as on the effectiveness of the health-care system as a whole. ${ }^{[8]}$ Although a national benchmark for the outpatient management of isolated anemia has not yet been set, it is relevant to comment on the results in Table 2 . In addition to the finding that the mean wait time between initial referral to hematology and subsequent GIM appointment was 63 days, it is encouraging to see that those patients with a hemoglobin level that categorized them as having a more severe anemia had shorter wait-times than those categorized as having a less severe anemia. These results reflect positively on the ability of the referral process to appropriately triage patients based on the severity of their anemia.

It is often unclear when a patient should be referred to a specialist and even more unclear about which specialty a specific patient should be referred to. In this study, we found that in addition to working up a discrete condition (anemia), the general internist also addressed non-anemia related aspects of care, such as changes to blood pressure medication or referral for a sleep study. The number of such changes for any given patient was shown to increase with the number of comorbidities identified at the initial appointment (see Table 4). A positive relationship between quality of care and number of chronic comorbid diseases has been previously described, particularly when care is provided by a generalist as opposed to a subspecialist. ${ }^{[9]}$ Though the focus of the present study is not on the quality of care being provided for unrelated comorbidities, the finding that many patients received non-anemia related adjustments to care provides insight into the broad scope of care a patient receives when seen by a generalist. The likelihood of more general care being provided increased as the number of comorbidities increased, which demonstrates the ability of the generalist to manage a discrete medical issue in the context of medical complexity. A limitation of this study is that we did not follow a comparable group of patients referred to hematology which were actually seen by the hematology service. Going forward, it would be reasonable to consider a randomized study in which patients referred for assessment of anemia were randomized between assessment by a generalist and by a hematologist.
Only $5 \%$ of the study population was referred back to hematology $(n=5)$. Of these, one was referred for a non-anemia related hematologic issue (thrombosis), three were referred for further investigation of a primary hematologic problem as the cause of the anemia, and one was referred back by the primary care physician after being seen by GIM, due to refractory anemia. The findings from the sub-group analysis on outcome of anemia severity demonstrated that the majority of patients improved a category level in anemia severity by the end of the 18 month follow up period. Taken together, these two points provide further evidence that patients are appropriately and safely managed by GIM, with minimal need to be re-referred to hematology.

It is not unexpected that a large proportion of patients with microcytic anemia were referred to GI. This likely reflects the need for GI assessment of patients with iron deficiency anemia, suggesting that the referring physician may consider referring such patients directly to GI. For the purposes of this study, a referral to GI was counted only if the referral was initiated by GIM, and therefore does not include those patients whose primary care providers had already referred them to GI or who had seen GI prior to their GIM appointment. This may account for the seemingly low proportion of patients referred to GI overall, given that iron deficiency is the leading cause of anemia both globally and in this cohort of patents. It is also relevant to note that this cohort consisted of a predominately middle-aged population, with the median age being 60 years. The higher incidence of colorectal cancer in this age group, a common etiology of iron deficiency anemia, is a possible explanation for this referral pattern and indeed reflects an appropriate practice. Additionally, given that the cohort had a female predominance $(62 \%)$, we would have anticipated seeing a higher rate of referral to gynecology. However, this too can be at least partially accounted for by the age of the cohort. It is possible that a younger population may have a higher incidence of referrals to gynecology, as alternate etiologies such as abnormal uterine bleeding, are more prevalent in this demographic.

With respect to work-up of the cause of anemia, only $5 \%$ of patients had a bone marrow biopsy performed. With the exception of two patients, these were all performed on patients re-referred to hematology for investigation of a primary hematologic issue. Bone marrow biopsy is uncommonly the first investigation performed when working up an isolated anemia, and this reflects the fact that the most common etiology of anemia is iron deficiency, for which blood work is most often sufficient for diagnosis. Again, comparative data about the practices of GIM and hematology were not available for the purposes of the study, so how this practice differs (if at all) from the work-up a hematologist would 
undertake is unclear.

To conclude, due to increasing demand for all medical services, and the ever-present challenge to meet these demands with limited clinic resources, patients in Nova Scotia who are referred by primary care for the work-up of isolated anemia have been referred for assessment and management by GIM - a practice that has been in place since 2012. We conclude that our results suggest that patients seen by GIM for the work-up and management of isolated anemia are evaluated in an efficient and effective manner, and may even have additional benefits, with respect to receiving more generalized care concerning their comorbid conditions. A future area of research could include a comparison between patients seen by hematology versus GIM, as it would further detail how the practices followed by both parties differ, and the potential impact it has on patient care.

\section{ACKNOWLEDGEMENTS}

Funding for data analysis with support from the NSHA Department of Medicine. Support for this research project is attributed to a summer studentship with funding provided by the Department of Medicine University Internal Medicine Research Foundation (UIMRF) Summer Studentship.

\section{CONFLiCTS OF INTEREST Disclosure}

The authors declare they have no conflict of interest.

\section{REFERENCES}

[1] World Health Organization. Worldwide Prevalence of Anaemia 19932005: WHO Global Database on Anaemia. 2008.

[2] Kassebaum NJ, Jasrasaria R, Naghavi M, et al. A systematic analysis of global anemia burden from 1990 to 2010. Blood. 2014 Jan 30; 123(5): 615-24. https://doi.org/10.1182/blood-2 013-06-508325

[3] Wu AW, Young Y, Skinner EA, et al. Quality of care and outcomes of adults with asthma treated by specialists and generalists in managed care. Arch Intern Med. 2001 Nov 26; 161(21): 255460. PMid: 11718586. https://doi.org/10.1001/archinte.1 61.21 .2554

[4] Go AS, Rao RK, Dauterman KW, et al. A systematic review of the effects of physician specialty on the treatment of coronary disease and heart failure in the United States. Am J Med. 2000 Feb 15; 108(3): 216-26. https://doi.org/10.1016/S0002-9 343 (99) 00430-1

[5] Yelin EH, Such CL, Criswell LA, et al. Outcomes for persons with rheumatoid arthritis with a rheumatologist versus a non- rheumatologist as the main physician for this condition. Med Care. 1998 Apr; 36(4): 513-22. PMid: 9544591. https ://doi .org/10 .1097/00005650-199804000-00007

[6] Linzer M, Myerburg RJ, Kutner JS, et al. Exploring the generalistsubspecialist interface in internal medicine. Am J Med. $2006 \mathrm{Jun}$; 119(6): 528-37. https://doi.org/10.1016/j.amjmed.2006. 03.007

[7] Smetana GW, Landon BE, Bindman AB, et al. A comparison of outcomes resulting from generalist vs specialist care for a single discrete medical condition: a systematic review and methodologic critique. Arch Intern Med. 2007 Jan 8; 167(1): 10-20. https: //doi.org/10.1001/archinte.167.1.10

[8] Wait Time Alliance. A Report Card on Wait Times in Canada. 2014.

[9] Higashi T, Wenger NS, Adams JL, et al. Relationship between number of medical conditions and quality of care. N Engl J Med. 2007 Jun 14; 356(24): 2496-504. https://doi .org/10.1056/NEJMsa 066253 